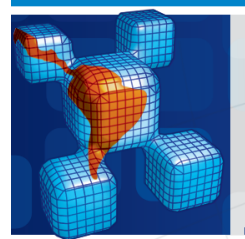

\title{
Vibration attenuation and shape control of surface mounted, embedded smart beam
}

\begin{abstract}
Active Vibration Control (AVC) using smart structure is used to reduce the vibration of a system by automatic modification of the system structural response. AVC is widely used, because of its wide and broad frequency response range, low additional mass, high adaptability and good efficiency. A lot of research has been done on Finite Element (FE) models for AVC based on Euler Bernoulli Beam Theory (EBT). In the present work Timoshenko Beam Theory (TBT) is used to model a smart cantilever beam with surface mounted sensors / actuators. A Periodic Output Feedback (POF) Controller has been designed and applied to control the first three modes of vibration of a flexible smart cantilever beam. The difficulties encountered in the usage of surface mounted piezoelectric patches in practical situations can be overcome by the use of embedded shear sensors / actuators. A mathematical model of a smart cantilever beam with embedded shear sensors and actuators is developed. A POF Controller has been designed and applied to control of vibration of a flexible smart cantilever beam and effect of actuator location on the performance of the controller is investigated. The mathematical modeling and control of a Multiple Input multiple Output (MIMO) systems with two sensors and two actuators have also been considered.

Keywords

AVC, FE, EBT, TBT, POF, MIMO, LTI, OLR, CLR
\end{abstract}

\section{Vivek Rathi and Arshad Hussain Khan}

Mechanical Engineering Department, Aligarh Muslim University, Aligarh India, 202001

\section{INTRODUCTION}

Undesired noise and vibrations have always been a major problem in many human activities and domains. From buildings to atomic force microscopes, all can be disturbed in their normal functions by vibrations and noise. Recent technological advancements such as the availability of high-power and low-cost computing, smart materials, and advanced control techniques have led to a growing use of AVC systems. The implication of active control is that desirable performance characteristics can be achieved through flexible and clever strategies, whereby actuators excite the structure based on the structure's response measured by sensors. 
Umapathy and Bandyopadhyay[20] discussed the vibration control aspects of a smart flexible beam for a Single Input Single Output (SISO) case. Hanagud et al. [11] developed a FE model for an active beam based on EBT and applied optimal output feedback control. Hawang et al.[13] developed a FE model for vibration control of a laminated plate with piezoelectric sensors /and actuators. Crawley et al [9] have presented the analytical and experimental development of piezoelectric actuators as elements of intelligent structures. FE models of a structure containing distributed piezoelectric sensors / actuators can also be seen in [10, 24]. Detailed survey on various control algorithms used in active vibration control studies has been presented by Alkhatib and Golnaragi [4]. A detailed comparative studies of different control algorithms on active vibration control of smart beam has been presented in [14, 21]. Kumar and Narayanan [15] carried out optimal location studies of sensor-actuator pairs using Linear Quadratic Regulator (LQR). Li et al. [16] proposed an optimal design methodology for the placement of piezoelectric actuator/sensor pairs. Molter et al. [17] carried out control design analysis for flexible manipulators using piezoelectric actuators. In their paper GA technique is employed for optimization of placement and size of piezoelectric material for optimal vibration control. Optimal controller design for the location, size and feedback of sensor/actuators have been carried out in references [12, 23].

Chandrashekhara and Vardarajan [8] have presented a FE model of a piezoelectric composite beam using higher - order shear deformation theory. Aldraihem et al. [3] have developed a laminated cantilever beam model using EBT and TBT with piezoelectric layers. Abramovich [1] has presented analytical formulation and closed form solutions of composite beam with piezoelectric actuators using TBT. Narayan and Balamurugan [18] have presented finite element formulation for the active vibration control study of smart beams, plates and shells and the controlled response is obtained using classical and optimal control strategies. In the analyses mentioned above, the controlled response has been obtained based on extension mode actuation. There have been very few studies based on shear mode actuation and sensing for the analysis of active structures.

The idea of exploiting the shear mode of creating transverse deflection in beams (sandwiched type) was first suggested by Sun and Zhang [19]. A FE approach was used by Benjeddou et al [6] to model a sandwich beam with shear and extension piezoelectric elements. It was observed that the shear actuator is more efficient in rejecting vibration than the extension actuator for the same control effort. Aldraihem and Khdeir [2] proposed analytical models and exact solutions for beams with shear and extension piezoelectric actuators. The models are based on TBT and HOBT. Exact solutions are obtained by using the state - space approach. Azulay and Abramovich [5] studied the effects of actuator location and number of patches on the actuator's performance for various configurations of patches and boundary conditions under mechanical and/or electrical loads. 


\section{POF CONTROL}

A standard result in control theory says that the poles of a linear time invariant (LTI) controllable system can be arbitrarily assigned by state feedback. If the original system is time invariant and the linear combinations are also constrained to the time invariant, the design problem is to choose an appropriate matrix of feedback gains. The problem of pole assignment by piecewise constant output feedback with infrequent observation was studied by Chammas and Leondes [7] for LTI systems.

Consider the system

$$
\begin{aligned}
& \dot{x}=A x(t)+B u(t), \\
& y(t)=C x(t)
\end{aligned}
$$

Where $x \in \mathfrak{R}^{n}, u \in \mathfrak{R}^{m}, y \in \mathfrak{R}^{p}, A \in \mathfrak{R}^{n \times n}, B \in \mathfrak{R}^{n \times m}, C \in \mathfrak{R}^{p \times n}$

$A, B, C$ are constants matrices and it is assumed that the system $(A, B, C)$ is controllable, observable and stable. Assume that output measurements are available from system at time instants $t=k \tau=0,1,2, \ldots$ Now, construct a discrete LTI system from these output measurements at rate $1 / \tau$ (sampling interval of $\tau$ seconds), the system so obtained is called the $\tau$ system and is given by,

$$
\begin{aligned}
& x(k+1) \tau=\Phi_{\tau} x(k \tau)+\Gamma_{\tau} u(k \tau) \\
& y(k \tau)=C x(k \tau)
\end{aligned}
$$

Now, design an output injection gain matrix $G$ such that Eigen values of $\left(\Phi_{\tau}+G C\right)$ are inside the unit circle i.e., $\operatorname{eig}\left(\Phi_{\tau}+G C\right)<1$.

$$
\begin{aligned}
& u(t)=K_{l} y(k t), \\
& {[k \tau+l \Delta] \leq t<[k \tau+(l+1) \Delta], K_{l+N}=K_{l}}
\end{aligned}
$$

For $l=0$, 1, - . - . - N $N$, where an output sampling interval $\tau$ is divided in to $N$ subintervals of width $\Delta=\tau / N$, the hold function being assumed constant. To see the relationship between the gain sequence $\left\{K_{l}\right\}$ and closed loop behavior, let $\{\Phi, \Gamma, C\}$ be a new system and denote the system sampled at rate $1 / \Delta$ as the $\Delta$ system and collect the gain matrices $K_{l}$ in to one matrix. If $(\Phi, \Gamma)$ system is controllable and $\left(\Phi_{\tau}, C\right)$ is observable, one can first choose and output injection gain $G$ to place the eigen values of $\left(\Phi_{N}+G C\right)$ in the desired locations inside the unit circle and then compute the POF gain sequence $\left\{K_{l}\right\}$ such that,

$$
\Gamma K=G
$$

and $\rho\left(\Phi^{N}+G C\right)<1$ is satisfied, where $\rho$ is spectral radius.

Werner and Furuta [22] proposed the performance index so that $\Gamma K=G$ need not be forced exactly. This constraint is replaced by a penalty function, which makes it possible to enhance the closed loop performance by allowing slight deviations from the original design and at the same time improving behavior. The performance index is, 


$$
J(k)=\sum_{l=0}^{\infty}\left[\begin{array}{ll}
x_{l}^{T} & u_{l}^{T}
\end{array}\right]\left[\begin{array}{ll}
\bar{Q} & 0 \\
0 & R
\end{array}\right]\left[\begin{array}{l}
x_{l} \\
u_{l}
\end{array}\right]+\sum_{k=1}^{\infty}\left(x_{k N}-x_{k N}^{*}\right)^{T} \bar{P}\left(x_{k N}-x_{k N}^{*}\right)
$$

\section{FORMULATION}

\subsection{Surface Mounted Sensors and Actuators} moment act at each nodal point.

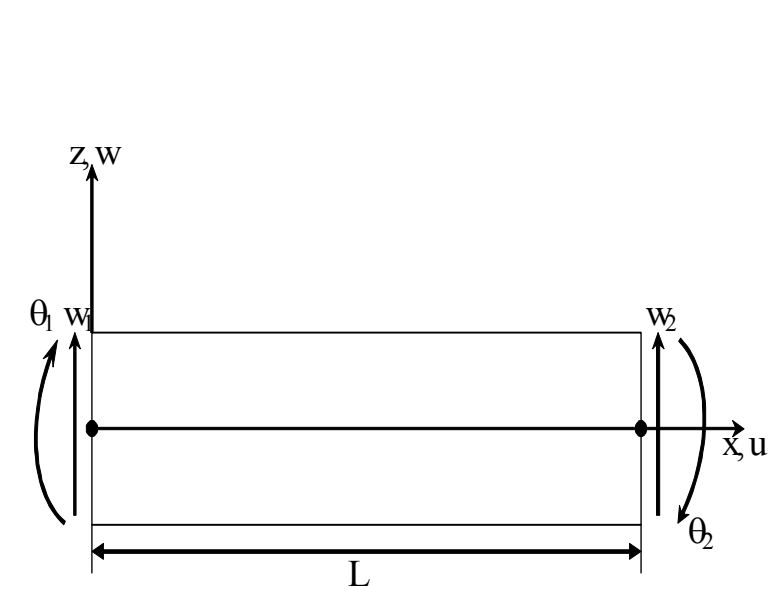

Figure 1 A Regular Beam Element

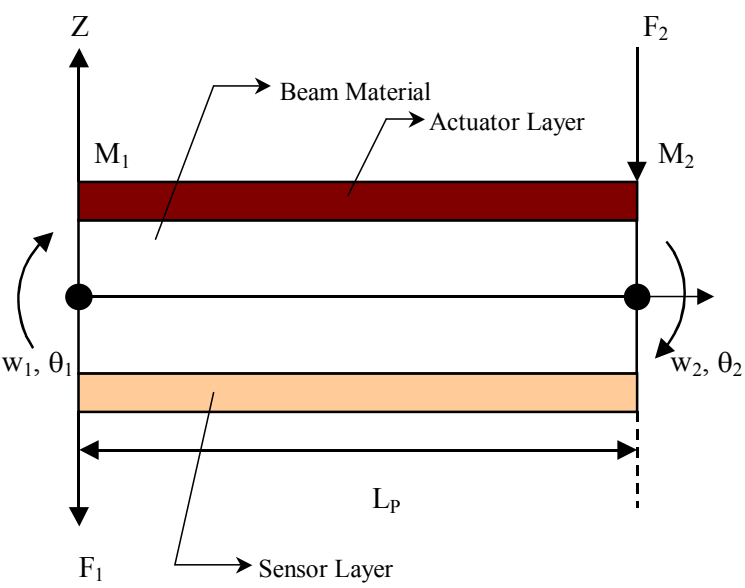

Figure 2 Piezoelectric Beam Elements with Sensors and Actuators

Where, $R \in \mathfrak{R}^{m \times n}, \bar{Q}$ and $\bar{P} \in \mathfrak{R}^{n \times n}$, are positive definite and symmetric weight matrices. The first term represents 'averaged' state and control energy whereas the second term penalizes deviation of $\mathrm{G}$.

The smart cantilever model is developed using a piezoelectric beam element, which includes sensor and actuator dynamics and a regular beam element based on TBT assumptions. The piezoelectric beam element is used to model the regions where the piezoelectric patch is bonded as sensor/actuator, and rest of the structure is modeled by the regular beam element.

The longitudinal axis of the regular beam element (Fig. 1), lies along the $\mathrm{X}$ - axis. The element has constant moment of inertia, modulus of elasticity, mass density and length. The element is assumed to have two degree of freedom, a transverse shear force and a bending

The displacement relation in the $\mathrm{x}, \mathrm{y}$ and $\mathrm{z}$ direction can be written as,

$$
\begin{aligned}
& u(x, y, z, t)=z \theta(x, t)=z\left(\frac{\partial w}{\partial x}-\beta(x)\right), \\
& v(x, y, z, t)=0 \\
& w(x, y, z, t)=w(x, t)
\end{aligned}
$$

Where,

$w$ is the time dependent transverse displacement of the centroidal axis, $\theta$ is the time dependent rotation of the cross - section about ' $\mathrm{Y}$ - axis'. 
For the static case with no external force acting on the beam, the equation of motion is,

$$
\begin{gathered}
\frac{\partial\left[\kappa G A\left(\frac{\partial w}{\partial x}+\theta\right)\right]}{\partial x}=0 \\
\frac{\partial\left(E I \frac{\partial \theta}{\partial x}\right)}{\partial x}-\kappa G A\left(\frac{\partial w}{\partial x}+\theta\right)=0
\end{gathered}
$$

The boundary conditions are given as,

At $x=0 \quad w=w_{1}, \quad \theta=-\theta_{1} \quad$ and $\quad$ At $\quad x=L \quad w=w_{2}, \quad \theta=-\theta_{2}$

The mass matrix is given by,

$$
\begin{gathered}
{[M]=\int_{0}^{L}\left[\begin{array}{c}
{\left[N_{w}\right]} \\
{\left[N_{\theta}\right]}
\end{array}\right]^{T}\left[\begin{array}{cc}
\rho A & 0 \\
0 & \rho I_{y y}
\end{array}\right]\left[\begin{array}{l}
{\left[N_{w}\right]} \\
{\left[N_{\theta}\right]}
\end{array}\right] d x} \\
{[M]=\left[M_{\rho A}\right]+\left[M_{\rho I}\right]}
\end{gathered}
$$

$\left[M_{\rho A}\right]$ in equation is associated with translational inertia and $\left[M_{\rho I}\right]$ is associated with rotary inertia, there expressions are given in the appendix.

The stiffness matrix is given by,

$$
[K]=\int_{0}^{L}\left[\begin{array}{c}
\frac{\partial}{\partial x}\left[N_{\theta}\right] \\
{\left[N_{\theta}\right]+\frac{\partial}{\partial x}\left[N_{w}\right]}
\end{array}\right]^{T}\left[\begin{array}{cc}
E I & 0 \\
0 & \kappa G A
\end{array}\right] \times\left[\begin{array}{c}
\frac{\partial}{\partial x}\left[N_{\theta}\right] \\
{\left[N_{\theta}\right]+\frac{\partial}{\partial x}\left[N_{w}\right]}
\end{array}\right] d x
$$

Finally we obtain;

$$
[K]=\frac{E I}{(1+\phi) L^{3}}\left[\begin{array}{cccc}
12 & 6 L & -12 & 6 L \\
6 L & (4+\phi) L^{2} & -6 L & (2-\phi) L^{2} \\
-12 & -6 L & 12 & -6 L \\
6 L & (2-\phi) L^{2} & -6 L & (4+\phi) L^{2}
\end{array}\right]
$$

Here $\phi$ is the ratio of the beam bending stiffness to the shear stiffness given by, $\phi=\frac{12}{L^{2}}\left(\frac{E I}{\kappa G A}\right)$,

$L$ is the length of beam element. $E$ is the Young's modulus of the beam material, $G$ is shear modulus of the beam material, $k$ is shear coefficient which depends on the material definition and cross - sectional geometry, $I$ is the moment of inertia of the beam element, $A$ is the area of cross - section of the beam element and $\rho$ is the mass density of the beam material.

The consistent force array is given as,

$$
\{F\}=\int_{0}^{L}\left[\begin{array}{c}
{\left[N_{w}\right]} \\
{\left[N_{\theta}\right]}
\end{array}\right]^{T}\left\{\begin{array}{c}
q \\
m
\end{array}\right\} d x .
$$

The piezoelectric element is obtained by sandwiching the regular beam element between two thin piezoelectric layers as shown in figure 2. The element is assumed to have two - structural degree of freedom at each nodal point and an electric degree of freedom. The piezoelectric 
layers are modeled based on EBT as the effect of shear is negligible and the middle steel layer is modeled based on TBT. The mass and stiffness matrix of piezoelectric layers is given by,

$$
\left[\mathrm{M}^{p}\right]=\frac{\rho_{p} A_{p} l_{p}}{420}\left[\begin{array}{cccc}
156 & 22 l_{p} & 54 & -13 l_{p} \\
22 l_{p} & 4 l_{p}^{2} & 13 l_{p} & -3 l_{p}^{2} \\
54 & 13 l_{p} & 156 & -22 l_{p} \\
-13 l_{p} & -3 l_{p}^{2} & -22 l_{p} & 4 l_{p}^{2}
\end{array}\right]\left[K^{p}\right]=\frac{E_{p} I_{p}}{l_{p}}\left[\begin{array}{cccc}
\frac{12}{l_{p}^{2}} & \frac{6}{l_{p}} & -\frac{12}{l_{p}^{2}} & \frac{6}{l_{p}} \\
\frac{6}{l_{p}} & 4 & -\frac{6}{l_{p}} & 2 \\
-\frac{12}{l_{p}^{2}} & -\frac{6}{l_{p}} & \frac{12}{l_{p}^{2}} & -\frac{6}{l_{p}} \\
\frac{6}{l_{p}} & 2 & -\frac{6}{l_{p}} & 4
\end{array}\right]
$$

$\rho_{p}$ is the mass density of piezoelectric beam element, $A_{p}$ is the area of piezoelectric patch $=2 t_{a} c, l p(=L)$ is the length of the piezoelectric patch. $E_{p}$ is the modulus elasticity of piezoelectric material, $I_{p}$ is the moment of inertia of piezoelectric layer w. r. t. the neutral axis of the beam

$$
I_{p}=\frac{1}{12} c t_{a}^{3}+c t_{a}\left[\frac{\left(t_{a}+t_{b}\right)}{2}\right]^{2}
$$

$t_{a}$ is the thickness of actuator, $t_{b}$ is the thickness of beam, $c$ is the width of beam.

The mass matrix for the piezoelectric beam element is given by,

$$
\left[M^{\text {piezo }}\right]=\left[M_{\rho A}\right]+\left[M_{\rho I}\right]+\left[M^{p}\right]
$$

Stiffness matrix $\left[K^{\text {piezo }}\right]$ for the piezoelectric beam element,

$$
\left[K^{\text {piezo }}\right]=[K]+\left[K^{p}\right]
$$

\subsubsection{Piezoelectric Strain Rate Sensors and Actuators}

The linear piezoelectric coupling between the elastic field and the electric field can be expressed by the direct and converse piezoelectric equations, respectively,

$$
D=d \sigma+e^{T} E_{f} \quad \varepsilon=s^{E} \sigma+d E_{f}
$$

$\sigma$ is the stress, $\epsilon$ is the strain, $\mathrm{E}^{f}$ is the electric field, e is the permittivity of the medium, $\mathrm{S}^{E}$ is the compliance of the medium, $\mathrm{d}$ is the piezoelectric constants.

Sensor Equation: If the poling is done along the thickness direction of the sensors with the electrodes on the upper and lower surfaces, the electric displacement is given by,

$$
D_{z}=d_{31} \times E_{p} \varepsilon_{x}=e_{31} \varepsilon_{x}
$$

e 31 is the piezoelectric stress / charge constants, $E_{p}$ is the Young's modulus of piezoelectric material, $\epsilon_{x}$ is the strain of the testing structure at a point.

The sensor output voltage is,

$$
V^{s}(t)=G_{c} e_{31} z c \int_{0}^{l_{p}} \mathrm{n}_{l}^{T} \dot{\mathrm{q}} \cdot d x
$$


$\mathrm{n}_{l}^{T}$ is the second spatial derivative of the shape function of the flexible beam and as a scalar vector product as,

$$
V^{s}(t)=\mathrm{p}^{T} \dot{\mathrm{q}}
$$

$\dot{\mathrm{q}}$ is the time derivative of the displacement vector, $\mathrm{p}^{T}$ is a constant vector.

The input voltage to an actuator is $V^{a}(t)$ given by,

$$
V^{a}(t)=K V^{s}(t)
$$

Actuator Equation: The strain developed by the electric field $\left(E_{f}\right)$ on the actuator layer is given by,

$$
\varepsilon_{A}=d_{31} E_{f}
$$

The control force applied by the actuator is,

$$
\mathrm{f}_{c t r l}=E_{p} d_{31} c \bar{z} \quad \int_{\mathrm{l}_{\mathrm{p}}} \mathrm{n}_{2} \cdot d x \cdot V^{a}(t)
$$

$\bar{z}=\frac{\left(t_{a}+t_{b}\right)}{2}$, is the distance between the neutral axis of the beam and the piezoelectric layer. Or as a scalar product as,

$$
\mathrm{f}_{c t r l}=\mathrm{h} \cdot V^{a}(t)
$$

$\mathrm{n}_{2}^{T}$ is the first spatial derivative of shape function of the flexible beam, $\mathrm{h}^{T}$ is a constant vector.

If any external forces described by the vector $\mathrm{f}_{e x t}$ are acting then, the total force vector becomes,

$$
\mathrm{f}^{t}=\mathrm{f}_{\text {ext }}+\mathrm{f}_{c t r l}
$$

\subsubsection{Dynamic Equation and State Space Model}

The dynamic equation of motion of the smart structure is finally given by,

$$
\begin{gathered}
\mathrm{M} \cdot \ddot{\mathrm{q}}+\mathrm{K} \cdot \mathrm{q}=\mathrm{f}_{e x t}+\mathrm{f}_{c t r l} \\
\mathrm{q}=\mathrm{T} \cdot \mathrm{g},
\end{gathered}
$$

$\mathrm{T}$ is the model matrix containing the eigen vectors representing the desired number of modes of vibration of the cantilever beam, $\mathbf{g}$ is the modal coordinate vector.

Equation (25) is then transformed in to,

$$
M^{*} \cdot \ddot{g}+C^{*} \cdot \dot{g}+K^{*} \cdot g=f_{\text {ext }}^{*}+f_{\text {ctrl }}^{*}
$$


$M^{*}$ is the generalized mass matrix $K^{*}$ is the generalized stiffness matrix, $C^{*}$ is the generalized damping matrix, $\mathrm{f}^{*}{ }_{\text {ext }}$ is the generalized external force vectors, $\mathrm{f}^{*}$ ctrl is the generalized control force vectors. The structural modal damping matrix is-:

$$
\mathrm{C}^{*}=\alpha \mathrm{M}^{*}+\beta \mathrm{K}^{*}
$$

$\alpha$ and $\beta$ are constants.

The state space model is

$$
\left[\begin{array}{c}
\dot{x}_{1} \\
\dot{x}_{2} \\
\dot{x}_{3} \\
\dot{x}_{4}
\end{array}\right]=\left[\begin{array}{cc}
0 & I \\
-M^{*-1} K^{*} & -M^{*-1} C^{*}
\end{array}\right]\left[\begin{array}{l}
x_{1} \\
x_{2} \\
x_{3} \\
x_{4}
\end{array}\right]+\left[\begin{array}{c}
0 \\
M^{*-1} T^{T} h
\end{array}\right] \mathrm{u}(t)+\left[\begin{array}{c}
0 \\
\mathrm{M}^{*-1} \mathrm{~T}^{T} \mathrm{f}
\end{array}\right] r(t)
$$

$\boldsymbol{u}(\boldsymbol{t})$ is the control input, $\boldsymbol{r}(\boldsymbol{t})$ is the external input to the system, $\boldsymbol{f}$ is the external force coefficient vector. The sensor equation for the modal state space form is given by;

$$
y(t)=\left[\begin{array}{lll}
0 & \mathrm{p}^{T} & \mathrm{~T}
\end{array}\right]\left[\begin{array}{l}
x_{1} \\
x_{2} \\
x_{3} \\
x_{4}
\end{array}\right]
$$

The above system may be represented as,

$$
\begin{gathered}
\dot{\mathrm{x}}=\mathrm{A} \cdot \mathrm{x}(\mathrm{t})+\mathrm{B} \cdot \mathrm{u}(\mathrm{t})+\mathrm{E} \cdot r(t) \\
y(t)=\mathrm{C}^{T} \mathrm{x}(\mathrm{t})
\end{gathered}
$$

\subsubsection{Validation for Surface Mounted Smart Beam}

To validate the present formulation and the computer program, a cantilever beam made of steel which is surface bonded with two PZT layers on both side is considered. The elastic modulus, poisons ratio and density of steel and PZT are 200GPa, 0.3 and $7500 \mathrm{Kg} / \mathrm{m}^{3}$ and $139 \mathrm{GPa}$, 0.3 and $7500 \mathrm{Kg} / \mathrm{m}^{3}$ respectively while the strain and stress constants of PZT are $23 \times 10^{-12}$ $\mathrm{m} / \mathrm{V}$ and 0.216 respectively [8]. The length, width and the thickness of the beam are $500 \mathrm{~mm}$, $30 \mathrm{~mm}$ and $2 \mathrm{~mm}$ respectively while the thickness of each of the PZT layers is $40 \mu \mathrm{m}$. The Voltages at the steel and PZT layers are set to zero. The beam is discretized into 20 elements to obtain converged results. The beam is excited with $0.2 \times 10^{-3} \mathrm{Ns}$ impulse load acting on the tip of the beam. The closed loop response of the tip displacement is obtained using constant gain negative velocity feedback (CGVF) control with gain $G_{v}=1$ and linear quadratic Regulator (LQR) control $\mathrm{Q}=10^{6}$ and $\mathrm{R}=1$ and compared with the response obtained under the same condition by the Narayanan and Balamurugan [8]. The control is applied after 0.5 seconds. The present response of the system is very well matched with the published results. Next, the 
first six open-loop and closed-loop natural frequencies of beam are presented in Table 1 and compared with reference [18]. These frequencies are in good agreement with the published results.

Table 1 First six natural frequencies of smart steel cantilever beam

\begin{tabular}{cccc}
\hline Open loop natural & $\begin{array}{c}\text { Closed loop natural } \\
\text { frequencies (Hz) } \\
\text { (CGVF control) }\end{array}$ & $\begin{array}{c}\text { Closed loop natural } \\
\text { frequencies }(\mathrm{Hz}) \\
\text { (LQR control) }\end{array}$ & $\begin{array}{c}\text { Natural } \\
\text { frequencies (HZ) } \\
{[8]}\end{array}$ \\
\hline 6.809 & 6.855 & 6.834 & 6.89 \\
41.64 & 43.19 & 41.69 & 43.285 \\
115.7 & 121.6 & 115.7 & 121.225 \\
226.2 & 237.5 & 226.3 & 237.72 \\
373.6 & 357.5 & 373.7 & 393.58 \\
557.8 & 506.3 & 557.9 & 589.63 \\
\hline
\end{tabular}
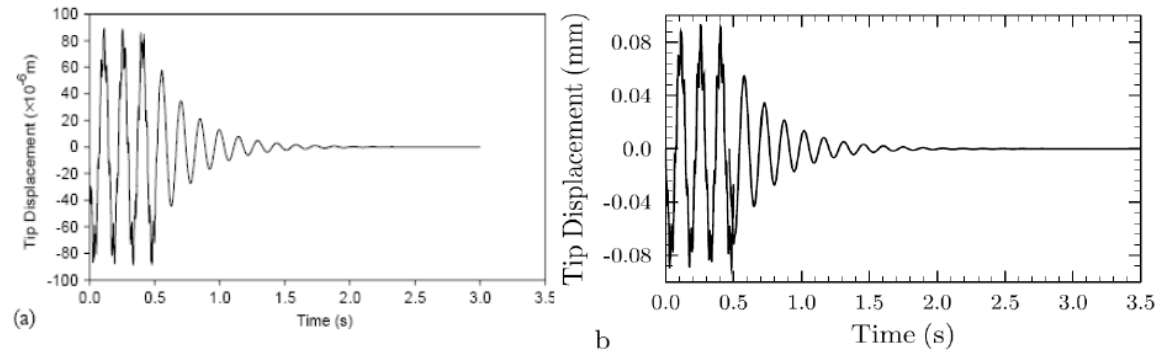

Figure 3 Closed loop response of smart cantilever steel beam (a) Narayanan and Balamurugan [18](reproduced with permission from Elsevier) and (b) Present obtained with negative CGVF control with Gv=1.
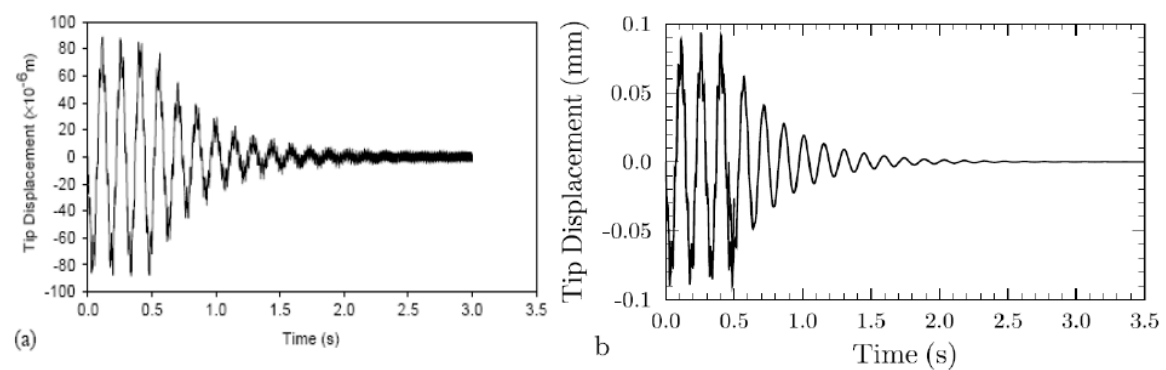

Figure 4 Closed loop response of smart cantilever steel beam (a) Narayanan and Balamurugan [18] (reproduced with permission from Elsevier) and (b) Present obtained with LQR control with $Q=10^{6}$ and $\mathbf{R}=\mathbf{1}$.

\subsection{Embedded Shear Sensors And Actuators}

The piezoelectric element is embedded on discrete locations of the sandwich beam as shown in Figure (5). The smart cantilever beam model is developed using a piezoelectric sandwich beam element, which includes sensor and actuator and a regular sandwiched beam element, which includes foam at the core. A FE model of a piezoelectric sandwich beam is developed using 
laminate beam theory. It consists of three layers. The assumption made is that the middle layer is perfectly glued to the carrying structure and the thickness of adhesive can be neglected and each layer behaves as a Timoshenko beam. The longitudinal axis of the sandwiched beam element lies along the $\mathrm{X}$ - axis. The element has constant moment of inertia, modulus of elasticity, mass density and length. The element is assumed to have three degree of freedom, a transverse shear force and a bending moment act at each nodal point.

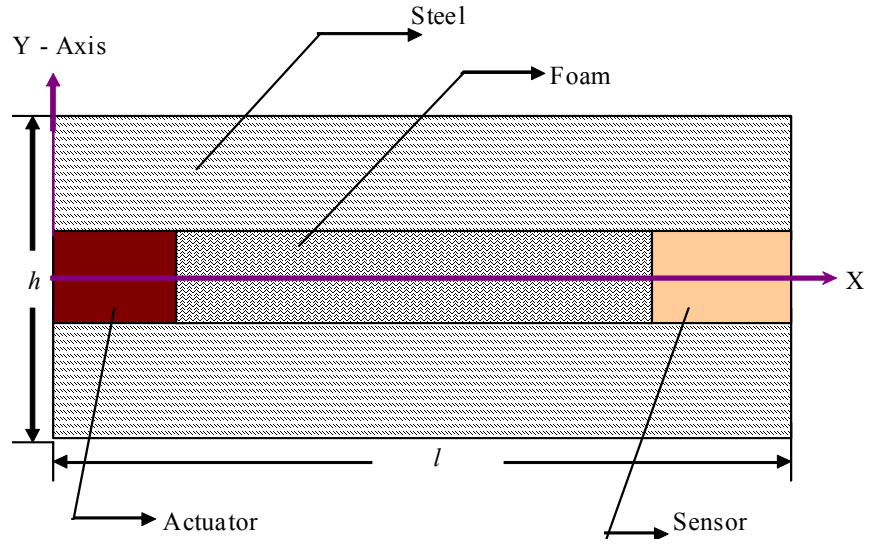

Figure 5 A Sandwiched Beam Element

The displacement relation of the beam $u(x, z)$ and $w u(x, z)$ can be written as,

$$
u(x, z)=u_{0}(x)-z \theta(x, t) \quad w(x, z)=w_{0}(x)
$$

$u_{0}(x)$ and $w_{0}(x)$ are the axial displacements of the point at the mid plane, $\theta(x)$ is the bending rotation of the normal to the mid plane.

The beam constitutive equation can be written as,

$$
\left[\begin{array}{l}
N_{x} \\
M_{x} \\
Q_{x z}
\end{array}\right]=\left[\begin{array}{ccc}
A_{11} & B_{11} & 0 \\
B_{11} & D_{11} & 0 \\
0 & 0 & A_{55}
\end{array}\right]\left[\begin{array}{c}
\frac{\partial u_{0}}{\partial x} \\
\frac{\partial \theta}{\partial x} \\
\theta+\frac{\partial w_{0}}{\partial x}
\end{array}\right]+\left[\begin{array}{c}
E_{11} \\
F_{11} \\
G_{55}
\end{array}\right]
$$

$A_{11}, B_{11}, D_{11}$ and $A_{55}$ are the extensional, bending and shear stiffness coefficients defined according to the lamination theory,

$$
\begin{aligned}
& A_{11}=c \sum_{k=1}^{N}\left(\bar{Q}_{11}\right)_{k}\left(z_{k}-z_{k-1}\right), \\
& B_{11}=c \sum_{k=1}^{N}\left(\bar{Q}_{11}\right)_{k}\left(z_{k}^{2}-z_{k-1}^{2}\right), \\
& D_{11}=\frac{c}{3} \sum_{k=1}^{N}\left(\bar{Q}_{11}\right)_{k}\left(z_{k}^{3}-z_{k-1}^{3}\right) \\
& A_{55}=c \kappa \sum_{k=1}^{N}\left(\bar{Q}_{55}\right)_{k}\left(z_{k}-z_{k-1}\right),
\end{aligned}
$$


$Z_{k}$ is the distance of the $k^{t h}$ layer from the $\mathrm{X}$ - axis, $\boldsymbol{N}$ is the number of layers, $\boldsymbol{k}$ is the shear correction factor usually taken equal to $5 / 6$.

The boundary conditions are given as,

At $x=0 \quad w=w_{1}, \quad \theta=\theta_{1}, \quad u=u_{1} \quad$ and $\quad$ At $\quad x=L \quad w=w_{2}, \quad \theta=\theta_{2}, \quad u=u_{2}$ After solving, we get,

$$
\begin{aligned}
& {[u]=\left[N_{u}\right]\left\{\begin{array}{c}
u_{1} \\
w_{1} \\
\theta_{1} \\
u_{2} \\
w_{2} \\
\theta_{2}
\end{array}\right\},[w]=\left[N_{w}\right]\left\{\begin{array}{c}
w_{1} \\
\theta_{1} \\
w_{2} \\
\theta_{2}
\end{array}\right\}, \theta=\left[N_{\theta}\right]\left\{\begin{array}{c}
w_{1} \\
\theta_{1} \\
w_{2} \\
\theta_{2}
\end{array}\right\},} \\
& {\left[N_{u}\right]=\left[\begin{array}{llllll}
N_{1} & N_{2} & N_{3} & N_{4} & N_{5} & N_{6}
\end{array}\right]} \\
& {\left[N_{W}\right]=\left[\begin{array}{llll}
N_{7} & N_{8} & N_{9} & N_{10}
\end{array}\right]} \\
& {\left[N_{\theta}\right]=\left[\begin{array}{llll}
N_{11} & N_{12} & N_{13} & N_{14}
\end{array}\right]}
\end{aligned}
$$

Values of $N_{1}$ to $N_{12}$ be given in the appendix,

The symmetric mass and stiffness matrices are given by,

$$
[M]=\left[\begin{array}{llllll}
M_{11} & M_{12} & M_{13} & M_{14} & M_{15} & M_{16} \\
M_{21} & M_{22} & M_{23} & M_{24} & M_{25} & M_{26} \\
M_{31} & M_{32} & M_{33} & M_{34} & M_{35} & M_{36} \\
M_{41} & M_{42} & M_{43} & M_{44} & M_{45} & M_{46} \\
M_{51} & M_{52} & M_{53} & M_{54} & M_{55} & M_{56} \\
M_{61} & M_{62} & M_{63} & M_{64} & M_{65} & M_{66}
\end{array}\right] \quad[K]=\left[\begin{array}{llllll}
K_{11} & K_{12} & K_{13} & K_{14} & K_{15} & K_{16} \\
K_{21} & K_{22} & K_{23} & K_{24} & K_{25} & K_{26} \\
K_{31} & K_{32} & K_{33} & K_{34} & K_{35} & K_{36} \\
K_{41} & K_{42} & K_{43} & K_{44} & K_{45} & K_{46} \\
K_{51} & K_{52} & K_{53} & K_{54} & K_{55} & K_{56} \\
K_{61} & K_{62} & K_{63} & K_{64} & K_{65} & K_{66}
\end{array}\right]
$$

Values of the mass and stiffness matrix coefficients are given in the appendix.

The material constants $\mathrm{Q}_{11}, \mathrm{Q}_{22}, \mathrm{Q}_{12}, \mathrm{Q}_{66}, \mathrm{G}_{13}$ and $\mathrm{G}_{23}$ for foam, steel and piezoelectric materials are given in table 2 . These constants are used to calculate the values of $\mathrm{A}_{11}, \mathrm{~B}_{11}$, $\mathrm{D}_{11}$ and $\mathrm{A}_{55}$

Table 2 Material Properties and Constants

\begin{tabular}{llll}
\hline Material Constants & Piezoelectric Material & Steel & Foam \\
\hline$G_{12}(M P a)$ & 24800 & 78700 & 99.9 \\
$G_{13}(M P a)$ & 24800 & 78700 & 99.9 \\
$G_{23}(M P a)$ & 24800 & 78700 & 99.9 \\
$d_{31}(m / V)$ & $-16.6 \times 10^{-9}$ & $\#$ & $\#$ \\
$d_{15}(m / V)$ & $1.34 \times 10^{-9}$ & $\#$ & $\#$ \\
$Q_{11}(M P a)$ & 68400 & 215000 & 85.4 \\
$Q_{22}(M P a)$ & 68400 & 215000 & 85.4 \\
$Q_{12}(M P a)$ & 12600 & 2880 & 75.8 \\
$Q_{66}(M P a)$ & 12600 & 78700 & 9.99 \\
\hline
\end{tabular}

Sensor Equation: The charge $q(t)$ accumulated on the piezoelectric electrodes is given by, 


$$
\mathrm{q}(t)=\iint_{A} D_{3} d A
$$

$\mathrm{D}_{3}$ is the electric displacement in the thickness direction, $A$ is the area of electrodes.

The current induced in sensor layer is converted in to the open circuit sensor voltage $V^{s}(t)$ using a signal-conditioning device with a gain of $G_{c}$ and applied to the actuator with the controller gain $K_{c}$.

$V^{s}(t)=G_{c} i(t), \quad V^{s}(t)=e_{15} c \frac{6 \eta}{\left(-12 \eta+l^{2}\right)}\left[\begin{array}{llllll}0 & 2 & -l & 0 & -2 & -l\end{array}\right][\dot{q}], \quad V^{s}(t)=[\mathrm{p}]^{T}[\dot{q}]$

$[\dot{q}]$ is the time derivative of the modal coordinate vector, $[\mathrm{p}]^{T}$ is a constant vector.

The input voltage of the actuator is $V^{a}(t)$, given by,

$$
V^{a}(t)=K_{c} V^{s}(t)
$$

Actuator Equation: The strain produced in the piezoelectric layer is directly proportional to the electric potential applied to the layer.

$$
\gamma_{x z} \propto E_{f}
$$

$\gamma_{x z}$ is the shear strain in the piezoelectric layer, $E_{f}$ is the electric potential applied to the actuator.

From the constitutive piezoelectric equation, we get,

$$
\gamma_{x z}=d_{15} E_{f}
$$

The shear force $Q_{x z}$ is given as

$$
Q_{x z}=c G d_{15} \frac{V^{a}(t)}{t_{p}} \int_{0}^{t_{p}} d z
$$

Or as a scalar product,

$$
\mathrm{f}_{c t r l}=\mathrm{h} \cdot V^{a}(t)
$$

Where, $\mathrm{f}_{c t r l}=Q_{x z}$ and $\mathrm{h}^{T}$ is a constant vector.

If any external forces described by the vector $\mathrm{f}_{c t r l}$ are acting, then total force vector becomes,

$$
\mathrm{f}^{t}=\mathrm{f}_{\text {ext }}+\mathrm{f}_{c t r l}
$$

Latin American Journal of Solids and Structures 1(2012) $1-25$ 


\subsubsection{Dynamic Equation and State Space Model:}

The dynamic equation is,

$$
\begin{gathered}
\mathrm{M}^{*} \cdot \ddot{\mathrm{g}}+\mathrm{C}^{*} \cdot \dot{\mathrm{g}}+\mathrm{K}^{*} \cdot \mathrm{g}=\mathrm{f}_{\mathrm{ext}}^{*}+\mathrm{f}_{\mathrm{ctrl}}^{*} \\
\mathrm{C}^{*}=\alpha \mathrm{M}^{*}+\beta \mathrm{K}^{*}
\end{gathered}
$$

The state space form of the system is obtained as,

$$
\left[\begin{array}{l}
\dot{x}_{1} \\
\dot{x}_{2} \\
\dot{x}_{3} \\
\dot{x}_{4} \\
\dot{x}_{5} \\
\dot{x}_{6}
\end{array}\right]=\left[\begin{array}{cc}
0 & I \\
-M^{*-1} K^{*} & -M^{*-1} C^{*}
\end{array}\right]\left[\begin{array}{l}
x_{1} \\
x_{2} \\
x_{3} \\
x_{4} \\
x_{5} \\
x_{6}
\end{array}\right]+\left[\begin{array}{c}
0 \\
M^{*-1} T^{T} h
\end{array}\right] \mathrm{u}(t)+\left[\begin{array}{c}
0 \\
M^{*-1} T^{T} f
\end{array}\right] r(t)
$$

The sensor equation for the modal state space form is given by;

$$
y(t)=\left[\begin{array}{lll}
0 & \mathrm{p}^{T} & \mathrm{~T}
\end{array}\right]\left[\begin{array}{l}
x_{1} \\
x_{2} \\
x_{3} \\
x_{4} \\
x_{5} \\
x_{6}
\end{array}\right]
$$

The above system can be represented as,

$$
\begin{gathered}
\dot{\mathrm{x}}=\mathrm{A} \cdot \mathrm{x}(\mathrm{t})+\mathrm{B} \cdot \mathrm{u}(\mathrm{t})+\mathrm{E} \cdot r(t) \\
y(t)=\mathrm{C}^{T} \mathrm{x}(\mathrm{t})
\end{gathered}
$$

\section{SIMULATION}

\subsection{Simulation of Surface Mounted Sensors and Actuators}

The state space representation of the cantilever beam with the surface mounted sensor / actuator is obtained by using nine regular beam elements and one piezoelectric element as shown figure (6).

The dimensions and properties of the flexible beam and piezoelectric sensor / actuator used in the numerical simulation are given in tables 3 and 4 respectively.

Two state space models of the smart cantilever beam have been obtained by keeping the AR 8 and 15, the length of the beam is kept constant and the thickness of the beam is varied. 


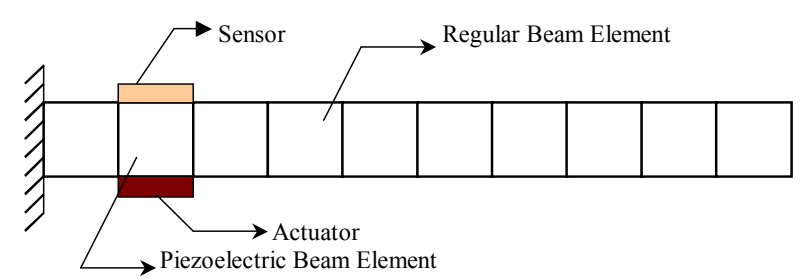

Figure 6 Cantilever Beam with Surface Mounted Sensors and Actuators

Table 3 Physical Properties of Steel Beam

\begin{tabular}{lll}
\hline Total length of beam $(\mathrm{mm})$ & $l$ & 500 \\
Width $(\mathrm{mm})$ & $\mathrm{b}$ & 24 \\
Young's modulus $(\mathrm{Pa})$ & $E_{b}$ & $193 \times 10^{9}$ \\
Density $\left.\mathrm{Kg} / \mathrm{m}^{3}\right)$ & $\rho_{b}$ & 8030 \\
Constants used in $\mathrm{C}^{*}$ & $\alpha, \beta$ & $10^{-3}, 10^{-4}$ \\
\hline
\end{tabular}

The POF control technique is used to design a controller to suppress vibration of a cantilever beam. For this purpose three vibration modes are considered. In the first case the AR is taken equal to 15. Configuration specifications of smart beam are as per table 3 and 4 . The collocated sensor and actuator are placed near the fixed end. The FE model of the surface mounted cantilever beam is developed in MATLAB using TBT. A sixth order space model of the system is obtained on retaining the first three modes of vibration of the system. The first three natural frequencies calculated are $106.03 \mathrm{~Hz}, 658.33 \mathrm{~Hz}, 1826.38 \mathrm{~Hz}$ respectively. An impulsive force of $10 \mathrm{~N}$ is applied for duration of $0.05 \mathrm{sec}$ and the open loop response (OLR) of the system is obtained as shown in figure 7. A controller based on the POF control algorithm has been designed to control the first three modes of vibration of the smart cantilever beam. The sampling interval used is $0.0004 \mathrm{sec}$. The sampling interval is divided in to 10 subintervals $(\mathrm{N}=10)$. The periodic output gain for the system is obtained by using the algorithm given for POF controller, the impulse response of the system with POF gain is shown in figure 8.

In the second case the AR is taken equal to 8, and again all other parameters are kept same as that in the first case for which $\mathrm{AR}$ is 15 . The first three natural frequencies calculated are $197.94 \mathrm{~Hz}, 1215.89 \mathrm{~Hz}$ and $3311.05 \mathrm{~Hz}$ respectively. The OLR and CLR (with POF gain) of the system is obtained as shown in figure 9 and 10.

Table 4 Properties of Piezoelectric Sensor /Actuator

\begin{tabular}{lll}
\hline Width $(\mathrm{mm})$ & $\mathrm{b}$ & 24 \\
Thickness $(\mathrm{mm})$ & $\mathrm{t}_{a}$ & 0.5 \\
Young's Modulus $(\mathrm{Pa})$ & $\mathrm{E}_{p}$ & $68 \times 10^{9}$ \\
Density $\left(\mathrm{Kg} / \mathrm{m}^{3}\right)$ & $\rho_{p}$ & 7700 \\
Piezoelectric strain Constant $\left(\mathrm{mV}^{-1}\right)$ & $\mathrm{d}_{31}$ & $125 \times 10^{-12}$ \\
Piezoelectric stress Constant $\left(\mathrm{VmN}^{-1}\right)$ & $\mathrm{e}_{31}$ & $10.5 \times 10^{-3}$ \\
\hline
\end{tabular}

Latin American Journal of Solids and Structures 1(2012) $1-25$ 


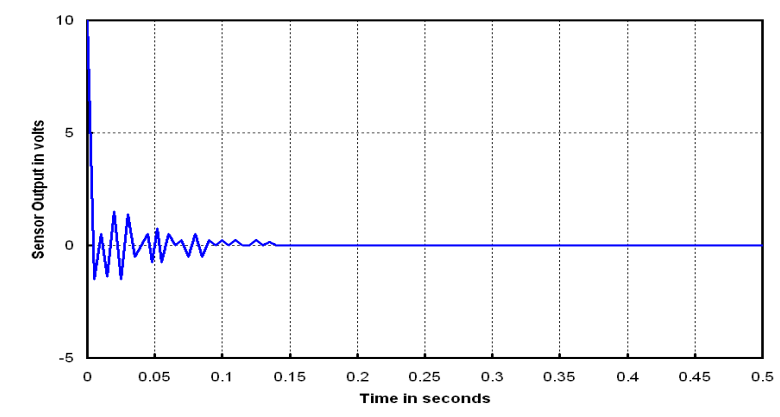

Figure 7 OLR of Surface Mounted Cantilever Beam with $A R=15$

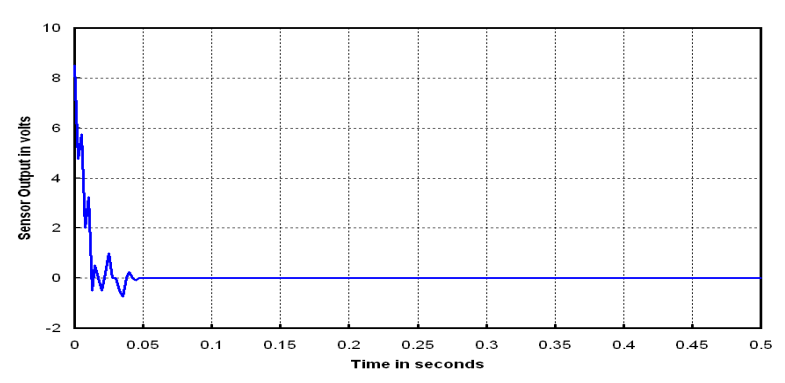

Figure 9 OLR of Surface Mounted Cantilever Beam with $A R=8$

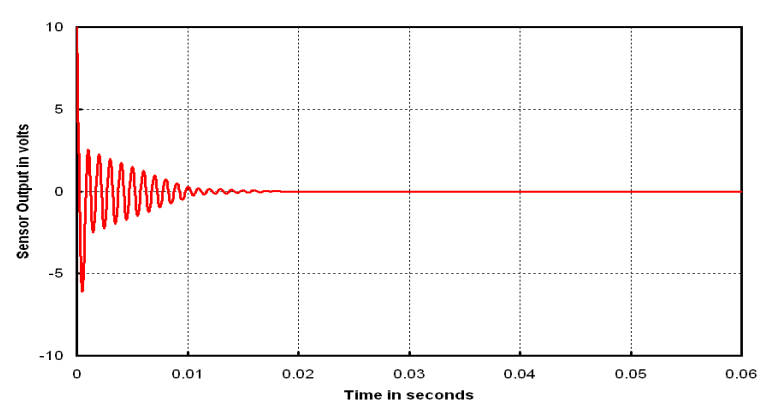

Figure 8 CLR of Surface Mounted Cantilever Beam with $A R=15$

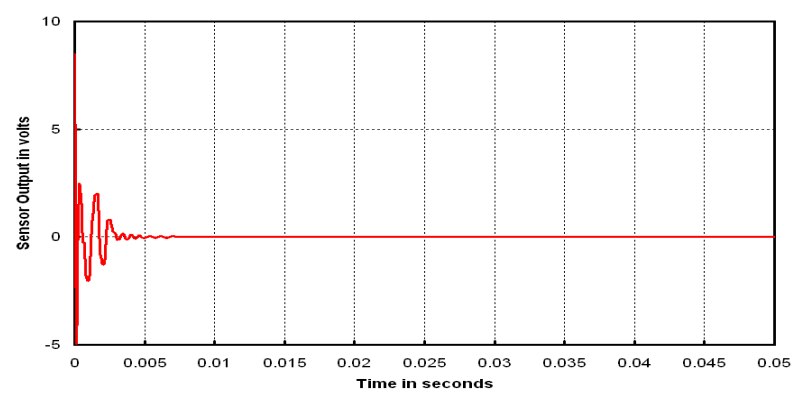
Figure $10 \begin{aligned} & \text { CLR of Surface Mounted Cantilever } \\ & \text { Beam with AR }=8\end{aligned}$

The variation of control signal with time is shown in figure 11 (for $\mathrm{AR}=15$ ) and 12 (for $\mathrm{AR}=8)$

A POF controller is designed for the Timoshenko beam models. Two cases $(A R=15,8)$ have been considered. By comparing the OLR and CLR in the first case for $A R=15$, we observed that there was a $89.43 \%$ decrease in settling time for the system after applying POF control, and a change of $89 \%$ in settling time was observed for the case with $A R=8$. Thus, it can be inferred from the simulation results, that a POF controller applied to a smart cantilever model based on TBT is able to satisfactorily control higher modes of vibration of the smart cantilever beam for a wide range of AR. 


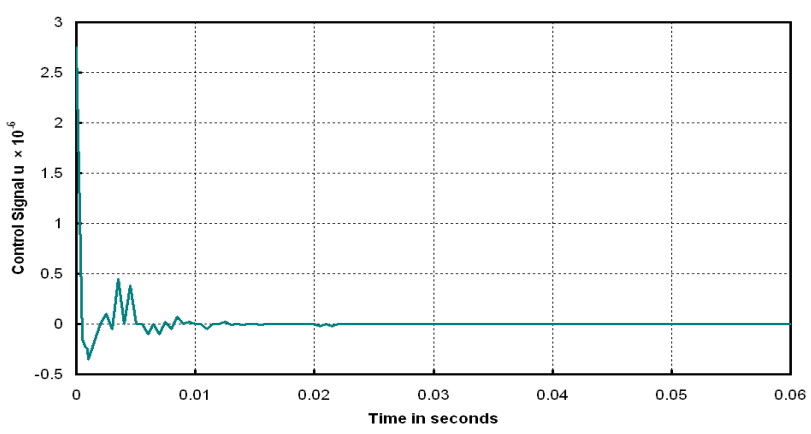

Figure 11 Control Signal of Surface Mounted Cantilever Beam with $\mathrm{AR}=15$

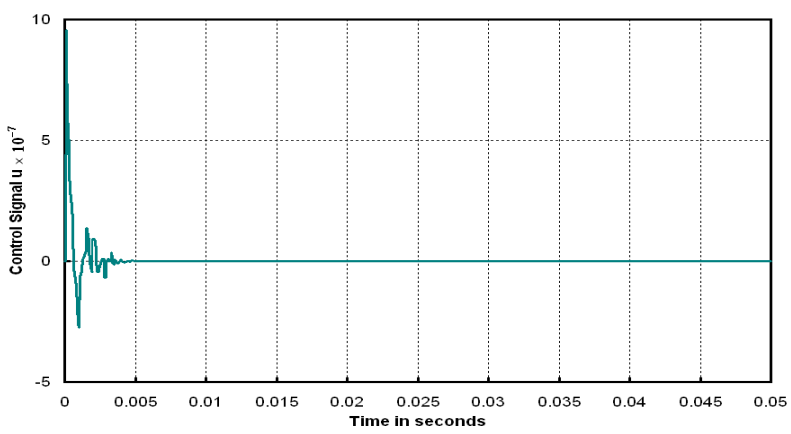

Figure 12 CLR of Surface Mounted Cantilever Beam with $A R=8$

\subsection{Simulation of Embedded Sensors and Actuators}

\subsubsection{Single Input Single Output (SISO) System}

The FE model of smart cantilever beam based on laminate beam theory is developed. Keeping the sensor location fixed and varying the position of the actuator, different state space models of the smart cantilever beam are obtained. A POF controller is designed to control the first three modes of vibration of the smart cantilever beam. Here an attempt has been made to find the optimum actuator position for a single input single output (SISO) system. Three cases have been considered.

In the first case FE model of the smart cantilever beam is obtained by dividing the beam into 10 elements. The actuator is placed as the $1^{\text {st }}$ element (at the fixed end) and the sensor is placed as the $8^{\text {th }}$ element as shown in figure 13. The length of beam is $200 \mathrm{~mm}$ and its cross - section is $10 \mathrm{~mm} \times 20 \mathrm{~mm}$. The length of piezoelectric patch is $200 \mathrm{~mm}$ and its cross - section is $6 \mathrm{~mm} \times 20 \mathrm{~mm}$. The material properties used for the generation of $\mathrm{FE}$ model are given in table 2. A ninth order space model of the system is obtained on retaining the first three modes of vibration of the system. The first three natural frequencies (same for all three models) are $44.9 \mathrm{~Hz}, 82.4 \mathrm{~Hz}$ and $131.5 \mathrm{~Hz}$ respectively.

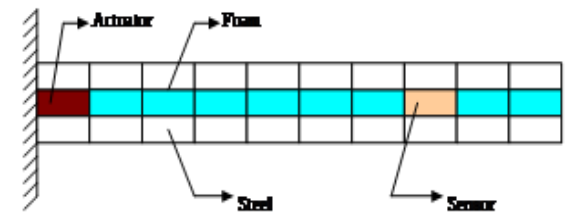

Figure 13 Smart Cantilever Beam with Actuator at $1^{\text {st }}$ Position and Sensor at $8^{\text {th }}$ Position

An impulsive force of $10 \mathrm{~N}$ is applied for duration of $0.05 \mathrm{sec}$ and the OLR of the system is obtained as shown in figure $14 \mathrm{~A}$ controller based on the POF control algorithm has been designed to control the first three modes of vibration of the smart cantilever beam. The sampling interval used is $0.07 \mathrm{~m} \mathrm{sec}$. The sampling interval is divided in to 10 subintervals ( $\mathrm{N}$ =10). The impulse response or CLR of the system with POF gain is shown in figure 15 . 


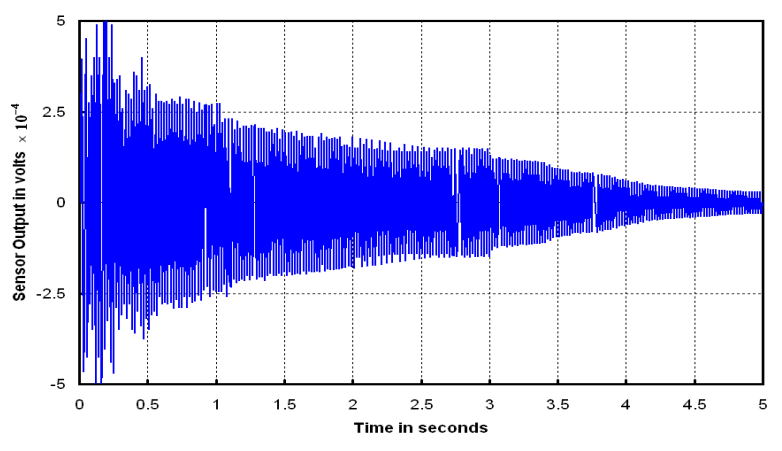

Figure 14 OLR of Smart Cantilever Beam with Actuator at $\mathbf{1}^{\text {st }}$ Position

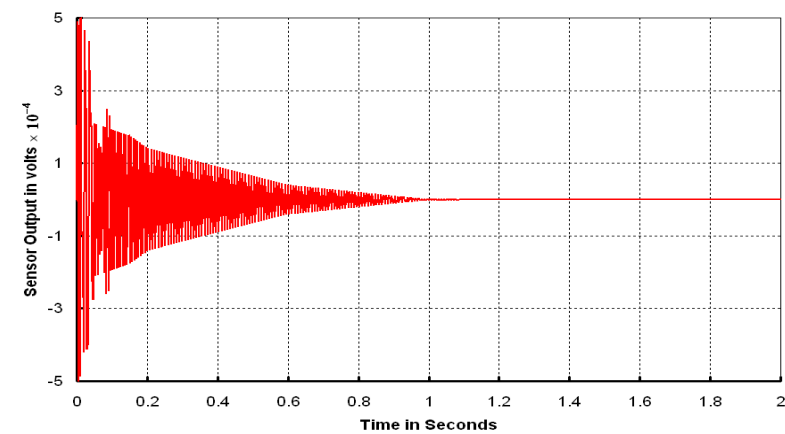

Figure 15 CLR of Smart Cantilever Beam with Actuator at $\mathbf{1}^{\text {st }}$ Position

In the second case, the actuator is placed as the $5^{\text {th }}$ element and the sensor is placed as the $8^{\text {th }}$ element as shown in figure 17. Other parameters are kept same.

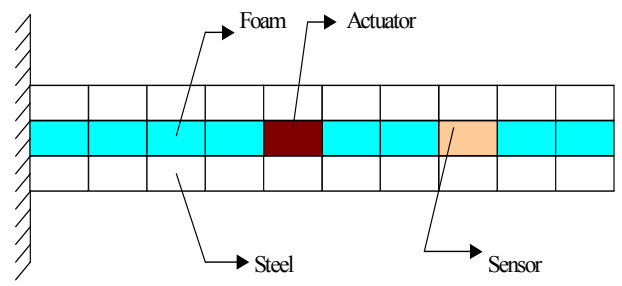

Figure 16 Smart Cantilever Beam with Actuator at $5^{\text {th }}$ Position and Sensor at $8^{\text {th }}$ Position

An impulsive force of $10 \mathrm{~N}$ is applied for duration of $0.05 \mathrm{sec}$ and the OLR of the system is obtained as shown in figure 17. The CLR of the system with POF gain is shown in figure 18.

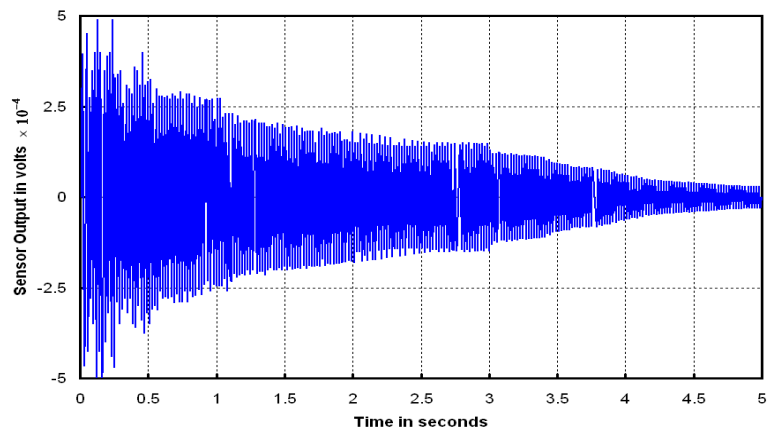

Figure 17 OLR of Smart Cantilever Beam with Actuator at $\mathbf{5}^{\text {th }}$ Position

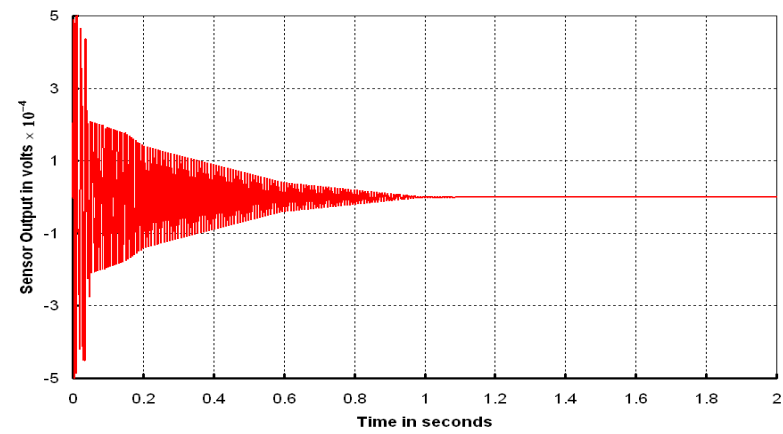

Figure 18 CLR of Smart Cantilever Beam with Actuator at $\mathbf{5}^{\text {th }}$ Position

In the third case, the actuator is placed as the $10^{\text {th }}$ element (at the free end) and the sensor is placed as the $8^{\text {th }}$ element as shown in figure 19. Other parameters are kept same as that of first case.

An impulsive force of $10 \mathrm{~N}$ is applied for duration of $0.05 \mathrm{sec}$ and the OLR of the system is 


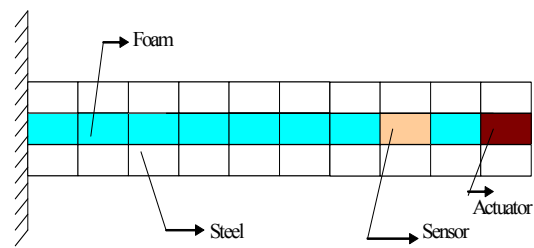

Figure 19 Smart Cantilever Beam with Actuator at $1^{\text {st }}$ Position and Sensor at $8^{\text {th }}$ Position

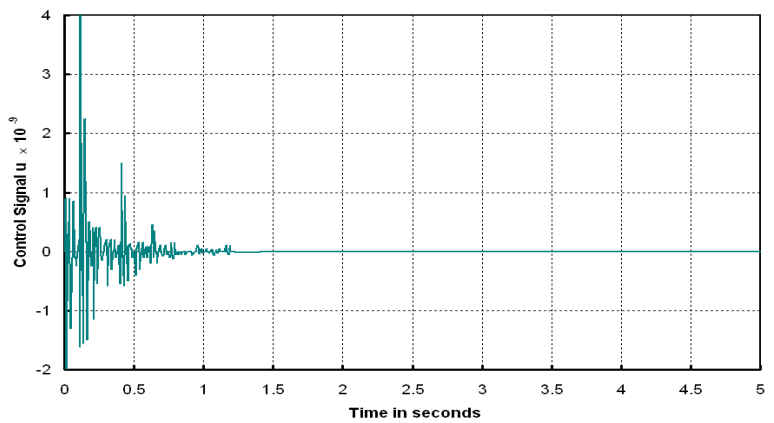

Figure 22 Control Signal of Smart Cantilever Beam with Actuator Placed at $1^{\text {st }}$ Position

Figure 20 OLR of Smart Cantilever Beam with Actuator at $10^{\text {th }}$ Position 24.

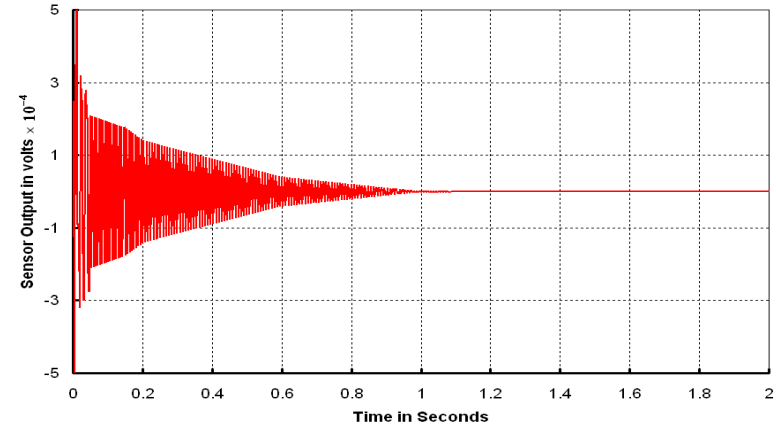

Figure 21 CLR of Smart Cantilever Beam with Actuator at $10^{\text {th }}$ Position

The variation of control signal with time for all the three cases are shown in figure 22 to

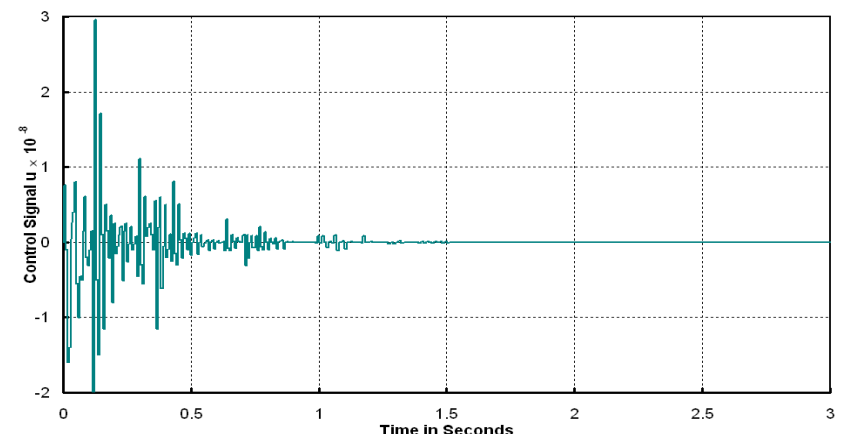

Figure 23 Control Signal of Smart Cantilever Beam with Actuator at $5^{\text {th }}$ Position

Here in the present case, the performance of the controller is evaluated for different actuator locations while the position of the sensor is kept constant. It can be inferred from the response characteristics that the actuator locations has negligible effect on the performance of the controller. 


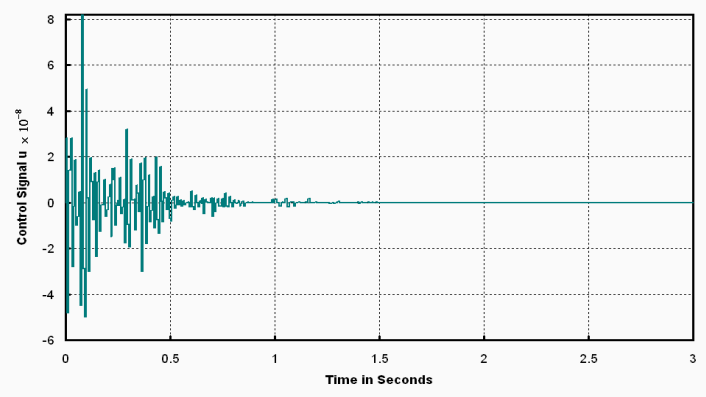

Figure 24 Control Signal of Smart Cantilever Beam with Actuator at $10^{\text {th }}$ Position

\subsubsection{Multi - Input Multi - Output (MIMO) Systems}

Active control of vibration of a smart cantilever beam through smart structure concept for a multivariable system (MIMO) case is considered here. The structure is modeled in space state from using Finite element method by dividing the beam in to $10 \mathrm{FE}$ and placing the sensors at the $6^{t h}$ and $10^{t h}$ positions and the actuators at the $4^{t h}$ and $8^{t h}$ position. Thus giving rise to MIMO with two actuator inputs $u_{1}$ and $u_{2}$ and two sensors outputs $y_{1}$ and $y_{2}$, The POF control technique is used to design a controller to suppress the first three modes of vibration of a smart cantilever beam for a multi variable system. The simulations are carried out in MATLAB. The parameters are kept same as that of the model used for SISO case. The first three natural frequencies calculated are $45.2 \mathrm{~Hz}, 83.2 \mathrm{~Hz}$ and $136.4 \mathrm{~Hz}$ respectively.

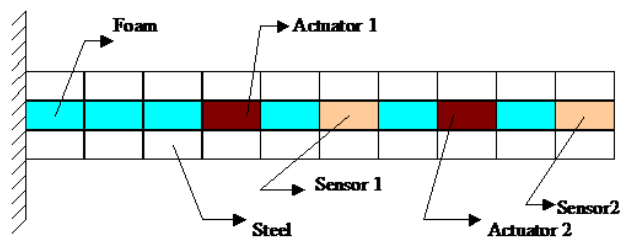

Figure 25 A MIMO Smart Cantilever Beam with Two Inputs and Two Outputs

An impulsive force of $10 \mathrm{~N}$ is applied for duration of $0.05 \mathrm{sec}$. A controller based on the POF control algorithm has been designed to control the first three modes vibration of the smart cantilever beam for the multivariable case. The CLR (sensor outputs $\mathrm{y}_{1}$ and $\mathrm{y}_{2}$ ) with periodic output feedback gain $K$ for the state space model of the system is shown in figure 27 and 31. Figures 29 and 33 show the variation of the control signal with time. 


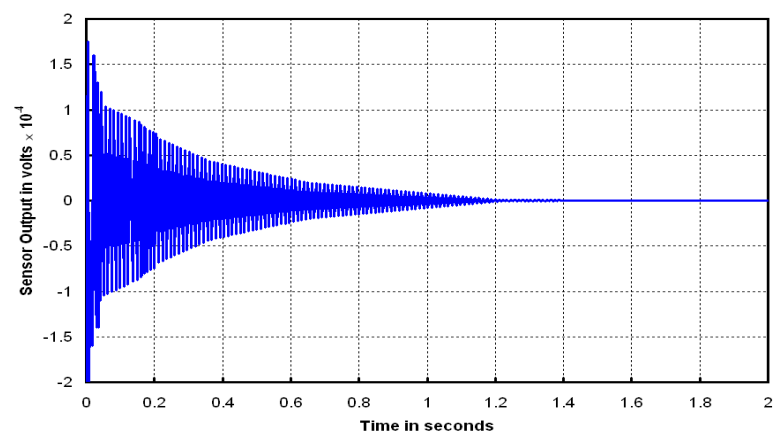

Figure 26 CLR of SISO System with Sensor at $6^{\text {th }}$ Position

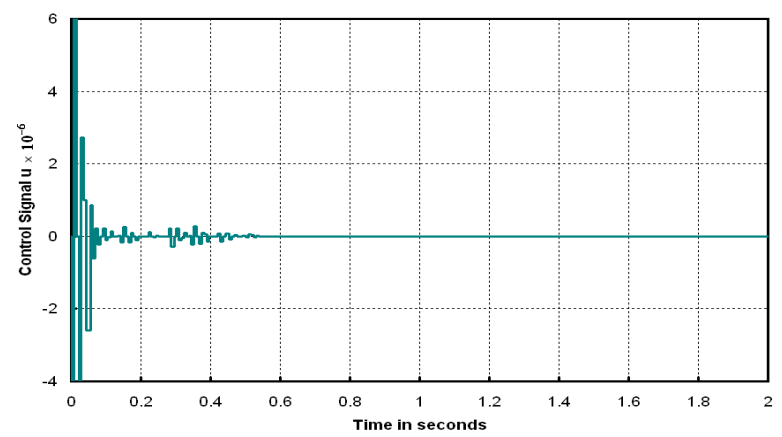

Figure 28 Control Input of SISO System with Sensor at $6^{\text {th }}$ Position and Actuator at $4^{\text {th }}$ Position

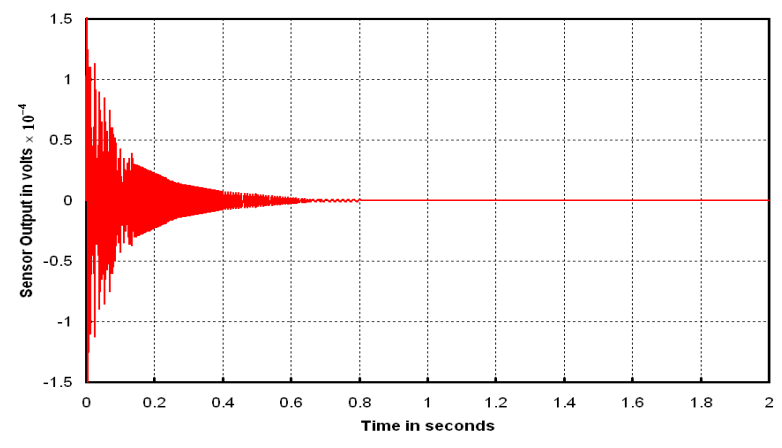

Figure 27 Response $\mathrm{y}_{1}$ of MIMO System

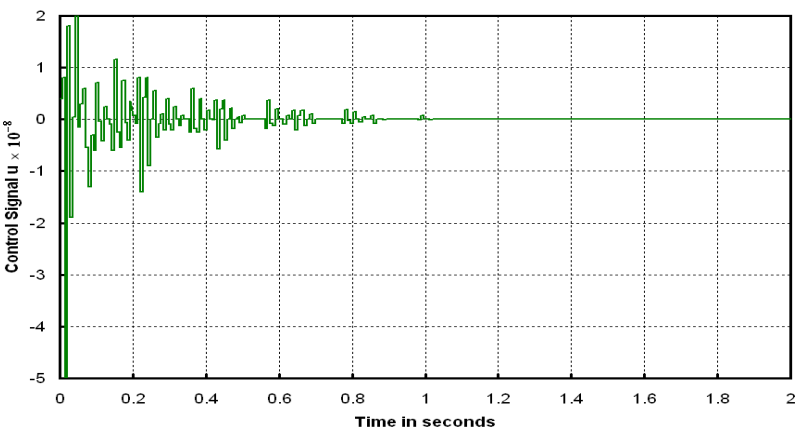

Figure 29 Control Input $u_{1}$ of MIMO System with POF

It can be inferred from the simulation results, that the system's performance meets the design requirements. It is also observed that the maximum amplitude of the sensor output voltage is less for the multivariable case and the response takes lesser time to settle. Controlling time is considerably reduced with MIMO systems as compared to SISO systems. 


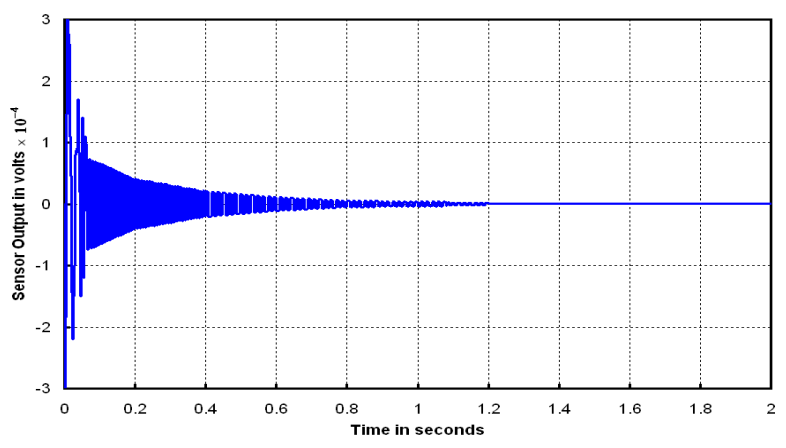

Figure 3030 CLR of SISO System with Sensor at $10^{\text {th }}$ Position and Actuator at $8^{\text {th }}$ Position

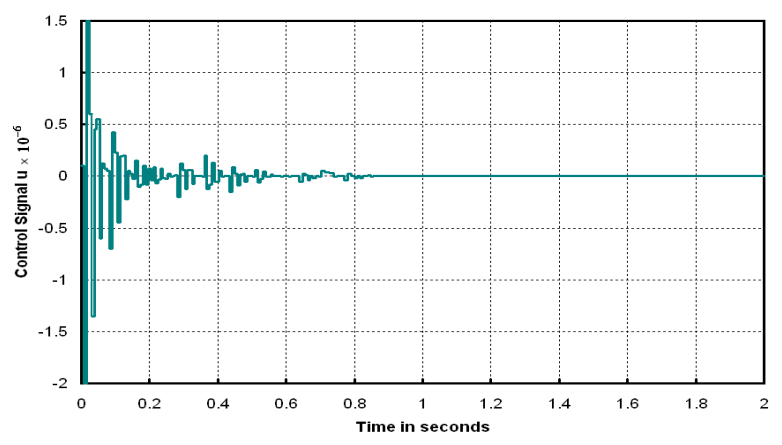

Figure 32 Control Input of SISO System with Sensor at $10^{t h}$ Position and Actuator at $8^{\text {th }}$ Position

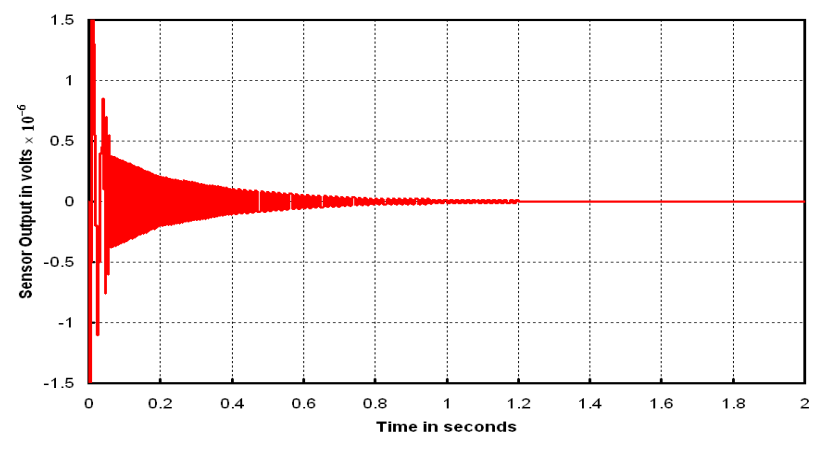

Figure 31 Response $\mathrm{y}_{2}$ of MIMO System with POF

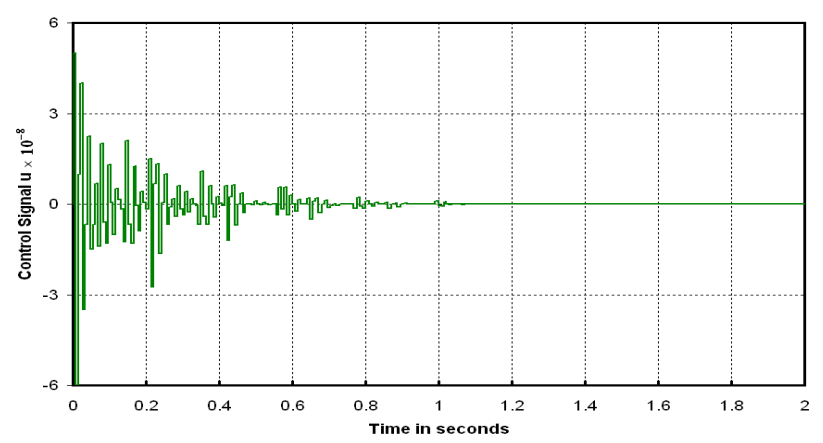

Figure 33 Control Input $u_{2}$ of MIMO System with POF

\section{CONCLUSION:}

An integrated FE model to analyze the vibration suppression capability of a smart cantilever beam with surface mounted piezoelectric devices based on TBT is developed. In practical situations a large number of modes of vibrations contribute to the structures response. In this work a FE model of a smart cantilever beam have been obtained by varying the AR from 8 to 15 , the length of the beam is kept constants and the thickness of the beam is varied. POF control technique is used to design a controller to suppress the vibration of the smart cantilever beam by considering three modes of vibration. Two different cases have been considered $(\mathrm{AR}=8,15)$. The simulation results show that the POF controller based on TBT is able to satisfactorily control the first three modes of vibration of the smart cantilever beam for different AR. Surface mounted piezoelectric sensors and actuators are usually placed at the extreme thickness positions of the structure to achieve most effective sensing and actuation. This subjects the sensors / actuators to high longitudinal stresses that might damage the piezoceramic material. Furthermore, surface mounted sensors/ actuators are likely to be damaged by contact with surrounding objects. Embedded shear sensors / actuators can be used to alleviate these problems. A FE model of a smart cantilever beam with embedded 

and the response takes lesser time to settle.

\section{APPENDIX}

1. Translational mass matrix:

2. Rotational Mass matrix

3. The mass matrix for the sandwich beam element,

Where,

iezoelectric shear sensors / actuators based on laminate theory is developed.

A POF controller is designed to control the vibration of the system. The performance of the controller is evaluated for different actuator locations while the position of sensor is kept constant. It was observed from the simulation results that the location of the actuator has negligible effect on the performance of the controller. A MIMO system with two sensors and two actuators has also been considered. A POF controller has been designed for the MIMO smart structure model to control the vibration of the system by considering the three modes of vibration. The beam with embedded shear sensors / actuators has been divided in to $10 \mathrm{FE}$ with the sensors placed at the $6^{\text {th }}$ and $10^{\text {th }}$ positions and the actuators placed at the $4^{\text {th }}$ and $8^{\text {th }}$ positions. It can be inferred from the simulation results, that when the system is placed with the controller, the system's performance meets the design requirements. It is observed that the maximum amplitude of the sensor output voltage is less for the multivariable case

$$
\left[M_{\rho A}\right]=\frac{\rho I}{210(1+\phi)^{2}}\left[\begin{array}{cccc}
{\left[70 \phi^{2}+147 \phi+78\right]} & {\left[35 \phi^{2}+77 \phi+44\right] \frac{L}{4}} & {\left[35 \phi^{2}+63 \phi+27\right]} & -\left[35 \phi^{2}+63 \phi+26\right] \frac{L}{4} \\
{\left[35 \phi^{2}+77 \phi+44\right] \frac{L}{4}} & {\left[7 \phi^{2}+14 \phi+8\right] \frac{L^{2}}{4}} & {\left[35 \phi^{2}+63 \phi+26\right] \frac{L}{4}} & -\left[7 \phi^{2}+14 \phi+6\right] \frac{L^{2}}{4} \\
{\left[35 \phi^{2}+63 \phi+27\right]} & {\left[35 \phi^{2}+63 \phi+26\right] \frac{L}{4}} & {\left[70 \phi^{2}+147 \phi+78\right]} & -\left[35 \phi^{2}+77 \phi+44\right] \frac{L}{4} \\
-\left[35 \phi^{2}+63 \phi+26\right] \frac{L}{4} & -\left[7 \phi^{2}+14 \phi+6\right] \frac{L^{2}}{4} & -\left[35 \phi^{2}+77 \phi+44\right] \frac{L}{4} & {\left[7 \phi^{2}+14 \phi+8\right] \frac{L^{2}}{4}}
\end{array}\right]
$$

$$
\left[M_{\rho I}\right]=\left[\begin{array}{cccc}
36 & -(15 \phi-3) L & -36 & -(15 \phi-3) L \\
-(15 \phi-3) L & \left(10 \phi^{2}+5 \phi+4\right) L^{2} & (15 \phi-3) L & \left(5 \phi^{2}-5 \phi-1\right) L^{2} \\
\left(10 \phi^{2}+5 \phi+4\right) L^{2} & (15 \phi-3) L & 36 & (15 \phi-3) L \\
-(15 \phi-3) L & \left(5 \phi^{2}-5 \phi-1\right) L^{2} & (15 \phi-3) L & \left(10 \phi^{2}+5 \phi+4\right) L^{2}
\end{array}\right]
$$

$$
[M]=\left[\begin{array}{llllll}
M_{11} & M_{12} & M_{13} & M_{14} & M_{15} & M_{16} \\
M_{21} & M_{22} & M_{23} & M_{24} & M_{25} & M_{26} \\
M_{31} & M_{32} & M_{33} & M_{34} & M_{35} & M_{36} \\
M_{41} & M_{42} & M_{43} & M_{44} & M_{45} & M_{46} \\
M_{51} & M_{52} & M_{53} & M_{54} & M_{55} & M_{56} \\
M_{61} & M_{62} & M_{63} & M_{64} & M_{65} & M_{66}
\end{array}\right]
$$

$$
M_{11}=\frac{1}{3} L I_{1}, M_{12}=M_{21}=\frac{1}{2} \frac{\gamma L^{2} I_{1}}{\left(12 \eta-\mathrm{L}^{2}\right)}, \quad M_{13}=M_{31}=-\frac{1}{4} \frac{\gamma L^{3} I_{1}}{\left(12 \eta-\mathrm{L}^{2}\right)}, \quad M_{14}=M_{41}=\frac{1}{6} L I_{1},
$$


350

351

$$
\begin{aligned}
& M_{15}=M_{51}=-\frac{1}{2} \frac{\gamma L^{2} I_{1}}{\left(12 \eta-\mathrm{L}^{2}\right)}, \quad M_{16}=M_{61}=-\frac{1}{4} \frac{\gamma L^{3} I_{1}}{\left(12 \eta-\mathrm{L}^{2}\right)}, \quad M_{24}=M_{42}=\frac{1}{2} \frac{\gamma L^{2} I_{1}}{\left(12 \eta-\mathrm{L}^{2}\right)}, \\
& M_{22}=\frac{1}{35} \frac{L\left(\begin{array}{c}
-294 I_{3} \eta L^{2}+35 I_{2} L^{3}+1680 I_{2} \eta^{2}+13 I_{3} L^{4}-420 I_{2} \eta L \\
+42 I_{1} L^{2}+42 \gamma^{2} I_{1} L^{2}
\end{array}\right)}{\left(12 \eta-\mathrm{L}^{2}\right)^{2}}, \\
& M_{23}=M_{32}=\frac{1}{210} \frac{L\left(\begin{array}{c}
11 I_{3} L^{5}-10080 I_{2} \eta^{2}+1260 I_{3} \eta^{2} L-231 I_{3} L^{3} \eta+126 \gamma^{2} I_{1} L^{3} \\
+1260 L I_{1} \eta+840 I_{2} \eta L^{2}+21 I_{1} L^{3}
\end{array}\right)}{\left(12 \eta-\mathrm{L}^{2}\right)^{2}}, \\
& M_{25}=M_{52}=\frac{3}{70} \frac{L\left(3 I_{3} L^{4}-28 \gamma^{2} I_{1} L^{2}-28 I_{1} L^{2}-84 I_{3} \eta L^{2}+560 I_{3} \eta^{2}\right)}{\left(12 \eta-L^{2}\right)^{2}}, \\
& M_{26}=M_{62}=\frac{1}{420} \frac{L\left(\begin{array}{c}
13 I_{3} L^{5}+10080 I_{2} \eta^{2}+2520 I_{3} \eta^{2} L-378 I_{3} L^{3} \eta+252 \gamma^{2} I_{1} L^{3} \\
-2520 L I_{1} \eta-840 I_{2} \eta L^{2}-42 I_{1} L^{3}
\end{array}\right)}{\left(12 \eta-\mathrm{L}^{2}\right)^{2}}
\end{aligned}
$$

$$
\begin{aligned}
& M_{55}=\frac{1}{35} \frac{L\left(\begin{array}{c}
13 I_{3} L^{4}-35 I_{2} L^{3}+42 \gamma^{2} I_{1} L^{2}+42 I_{1} L^{2}-294 I_{3} \eta L^{2} \\
+420 I_{2} \eta L+1680 I_{3} \eta^{2}
\end{array}\right)}{\left(12 \eta-\mathrm{L}^{2}\right)^{2}}, \\
& M_{56}=M_{65}=\frac{1}{210} \frac{L\left(\begin{array}{c}
11 I_{3} L^{5}+10080 I_{2} \eta^{2}+1260 I_{3} \eta^{2} L-231 I_{3} L^{3} \eta+126 \gamma^{2} I_{1} L^{3} \\
+1260 L I_{1} \eta-840 I_{2} \eta L^{2}+21 I_{1} L^{3}
\end{array}\right)}{\left(12 \eta-\mathrm{L}^{2}\right)^{2}} \\
& M_{66}=\frac{1}{210} \frac{L\left(\begin{array}{c}
252 I_{3} \eta^{2} L^{2}-42 I_{3} L^{4} \eta+10080 I_{1} \eta^{2}+63 \gamma^{2} I_{1} L^{4}+28 I_{1} L^{4} \\
-420 I_{1} \eta L^{2}+2 I_{3} L^{6}+2520 I_{2} \eta^{2} L-210 I_{2} \eta L^{3}
\end{array}\right)}{\left(12 \eta-\mathrm{L}^{2}\right)^{2}} .
\end{aligned}
$$

1. The stiffness matrix for the sandwich beam element is

$$
[K]=\left[\begin{array}{llllll}
K_{11} & K_{12} & K_{13} & K_{14} & K_{15} & K_{16} \\
K_{21} & K_{22} & K_{23} & K_{24} & K_{25} & K_{26} \\
K_{31} & K_{32} & K_{33} & K_{34} & K_{35} & K_{36} \\
K_{41} & K_{42} & K_{43} & K_{44} & K_{45} & K_{46} \\
K_{51} & K_{52} & K_{53} & K_{54} & K_{55} & K_{56} \\
K_{61} & K_{62} & K_{63} & K_{64} & K_{65} & K_{66}
\end{array}\right]
$$

$K_{11}=\frac{A A_{11}}{L}, K_{12}=K_{21}=\frac{A B_{11}}{L}, K_{13}=K_{31}=0, K_{14}=K_{41}=-\frac{A A_{11}}{L}, K_{15}=K_{51}=-\frac{A B_{11}}{L}$, $K_{16}=K_{61}=0, \quad K_{22}=-\frac{1}{10} \frac{A L\left(D_{11} L^{3}-10 B_{11} \gamma L^{2}+60 A_{55} L+60 \eta^{2} A_{11} L+120 \gamma B_{11} \eta\right)}{\left(12 \eta-L^{2}\right)^{2}}$,

$K_{23}=K_{32}=\frac{6}{5} \frac{A}{L} \frac{\left(D_{11} L^{4}+10 A_{55} L^{2}+10 \gamma^{2} A_{11} L^{2}-20 \eta D_{11} L^{2}+120 D_{11} \eta^{2}\right)}{\left(12 \eta-L^{2}\right)^{2}}$,

$K_{24}=K_{42}=-\frac{A B_{11}}{L}, K_{25}=K_{52}=-\frac{6}{5} \frac{A}{L} \frac{\left(D_{11} L^{4}+10 A_{55} L^{2}+10 \gamma^{2} A_{11} L^{2}-20 \eta D_{11} L^{2}+120 D_{11} \eta^{2}\right)}{\left(12 \eta-\mathrm{L}^{2}\right)^{2}}$,

$K_{26}=K_{62}=\frac{1}{10} \frac{A L\left(-D_{11} L^{3}-10 B_{11} \gamma L^{2}-60 A_{55} L-60 \gamma^{2} A_{11} L+120 \gamma B_{11} \eta\right)}{\left(12 \eta-L^{2}\right)^{2}}$,

$K_{33}=\frac{A\left(\begin{array}{c}2 L^{6} D_{11}-30 L^{4} D_{11} \eta+180 L^{2} D_{11} \eta^{2}-15 L^{5} \gamma B_{11}+2160 A_{55} \eta^{2} \\ +60 A_{55} L^{4}-360 A_{55} \eta L^{2}+180 \gamma L^{3} B_{11} \eta+45 \gamma^{2} L^{4} A_{11}\end{array}\right)}{15 L\left(12 \eta-L^{2}\right)^{2}}, K_{34}=K_{43}=0$, 
354

$$
\begin{aligned}
K_{35}= & K_{53}=\frac{1}{10} \frac{A L\left(D_{11} L^{3}-10 B_{11} \gamma L^{2}+60 A_{55} L+60 \gamma^{2} A_{11} L+120 \gamma B_{11} \eta\right)}{\left(12 \eta-L^{2}\right)^{2}}, K_{44}=\frac{A A_{11}}{L}, \\
K_{45}= & K_{54}=\frac{A B_{11}}{L}, K_{46}=K_{64}=0, \\
K_{36}= & K_{63}=\frac{-A\left(\begin{array}{c}
L^{6} D_{11}-60 L^{4} A_{55}-90 L^{4} A_{11} \gamma^{2}-60 L^{4} \eta D_{11}+360 D_{11} L^{2} \eta \\
+4320 A_{55} \eta^{2}-720 A_{55} \eta L^{2}
\end{array}\right)}{30 L\left(12 \eta-L^{2}\right)^{2}}, \\
K_{55}= & \frac{6}{5} \frac{A}{L} \frac{\left(D_{11} L^{4}+10 A_{55} L^{2}+10 \gamma^{2} A_{11} L^{2}-20 \eta D_{11} L^{2}+120 D_{11} \eta^{2}\right)}{\left(12 \eta-L^{2}\right)^{2}}, \\
K_{56}= & K_{65}=-\frac{1}{10} \frac{A L\left(-D_{11} L^{3}-10 B_{11} \gamma L^{2}-60 A_{55} L-60 \gamma^{2} A_{11} L+120 \gamma B_{11} \eta\right)}{\left(12 \eta-L^{2}\right)^{2}}, \\
K_{66}= & \frac{A\left(\begin{array}{c}
2 L^{6} D_{11}+15 L^{5} B_{11} \gamma-30 L^{4} D_{11} \eta+2160 A_{55} \eta^{2}+180 L^{2} D_{11} \eta^{2} \\
+60 A_{55} L^{4}-360 A_{55} \eta L^{2}-180 \gamma L^{3} B_{11} \eta+45 \gamma^{2} L^{4} A_{11}
\end{array}\right)}{15 L\left(12 \eta-L^{2}\right)^{2}} .
\end{aligned}
$$

\section{References}

[1] H. Abramovich. Deflection control of laminated composite beams with piezoceramic layers - close form solution. Composite Structures, 43(3):2217-231, 1998.

[2] O. J. Aldraihem and A. A. Khdeir. Smart beams with extension and thickness-shear piezoelectric actuators. Journal Of Smart Materials And Structures, 9(1):1-9, 2000.

[3] O.J. Aldraihem, R.C. Wetherhold, and T. Singh. Distributed control of laminated beams: Timoshenko vs. euler bernoulli theory. Journal of Intelligent Materials System and Structures, 8:149-157, 1997.

[4] R. Alkhatib and M.F. Golnaraghi. Active structural vibration control: a review. Shock and Vibration Digest, $35(5): 367-383,2003$.

[5] L.E. Azulay and H. Abramovich. piezoelectric actuation and sensing mechanisms - closed form solution. Composite Structures, 64(3):443-453

[6] A. Benjeddou, M.A. Trindade, and R. Ohayon. New shear actuated smart structure beam finite element. AIAA Journal, 37:378-383, 1999.

[7] A.B. Chammas and C.T. Leondes. Pole placement by piecewise constants output feedback. International Journal Of Control, 29(1):31-38, 1979.

[8] K. Chanrashekhara and S. Vardarajan. Adaptive shape control of composite beams with piezoelectric actuators. Journal of Intelligent Material Systems and Structures, 8:112-124, 1997.

[9] E.F. Crawley and J. De Luis. Use of piezoelectric actuators as elements of intelligent structures. AIAA Journal, $25: 1373-1385,1987$.

[10] C. Doschner and M. Enzmann. On model based controller design for smart structure. Smart Mechanical Systems Adaptronics International, pages 157-166.

[11] S. Hanagud, M.W. Obal, and A.J. Callise. Optimal vibration control by the use of piezoelectric sensors and actuators. Journal Of Guidance, Control and Dynamics, 15(5).

[12] Q. Hu and G. Ma. Variable structure maneuvering control and vibration suppression for flexible spacecraft subject to input nonlinearities. Smart Materials and Structures, 15(6):1899-1911, 2006.

[13] W. Hwang and H.C. Park. Finite element modeling of piezoelectric sensors and actuators. AIAA Journal, 31(5):930937, 1993.

[14] S. Kapuria and M.Y. Yasin. Active vibration control of piezoelectric laminated beams with electroded actuators and sensors using an efficient finite element involving an electric node. Smart Materials and Structures, $19,2010.045019$.

[15] K.R. Kumar and S. Narayanan. Active vibration control of beams with optimal placement of piezoelectric sensor/actuator pairs. Smart Mater. Struct, 17, 2008. 055008 (15pp). 
[16] Y. Li, J. Onoda, and K. Minesugi. Simultaneous optimization of piezoelectric actuator placement and feedback for vibration suppression. Acta Astronautica, 50.

[17] A. Molter, A. Otavio Alves da S., S. Jun Ono Fonseca, and V. Bottega. Simultaneous piezoelectric actuator and sensor optimization and control design of manipulators with flexible links using sdre method. Mathematical Problems in Engineering, 2010:23. Article ID 362437.

[18] S. Narayanana and V. Balamurugan. Finite element modelling of piezolaminated smart structures for active vibration control with distributed sensors and actuators. Journal of Sound and Vibration, 262:529-562, 2003.

[19] C.T. Sun and X.D. Zhang. Use of thickness shear mode in adaptive sandwich structures. Smart Materials and Structures, 4(3):205-207, 1995.

[20] M. Umapathy and B. Bandyopadhyay. Control of flexible beam through smart structure concept using periodic output. System Science, 26.

[21] C.M.A. Vasques and J.D. Rodrigues. Active vibration control of smart piezoelectric beams: Comparison of classical and optimal feedback control strategies. Computers and Structures, 84:1459-1470, 2006.

[22] H. Werner and K. Furuta. Simultaneous stabilization based on output measurements. Kybernrtca, 31(2):395-411.

[23] S. X. Xu and T.S. Koko. Finite element analysis and design of actively controlled piezoelectric smart structures. Finite Element in Analysis and Design, 40(3):241-262, 2004.

[24] M.Y. Yasin, N. Ahmad, and N. Alam. Finite element analysis of actively controlled smart plate with patched actuators and sensors. Latin American Journal of Solids and Structures, 7:227-247, 2010. 
